\title{
Medians and means in Finsler geometry
}

\author{
Marc Arnaudon and Frank Nielsen
}

\begin{abstract}
We investigate existence and uniqueness of $p$-means $e_{p}$ and the median $e_{1}$ of a probability measure $\mu$ on a Finsler manifold, in relation with the convexity of the support of $\mu$. We prove that $e_{p}$ is the limit point of a continuous time gradient flow. Under some additional condition which is always satisfied for $p \geq 2$, a discretization of this path converges to $e_{p}$. This provides an algorithm for determining the Finsler center points.
\end{abstract}

\section{Contents}

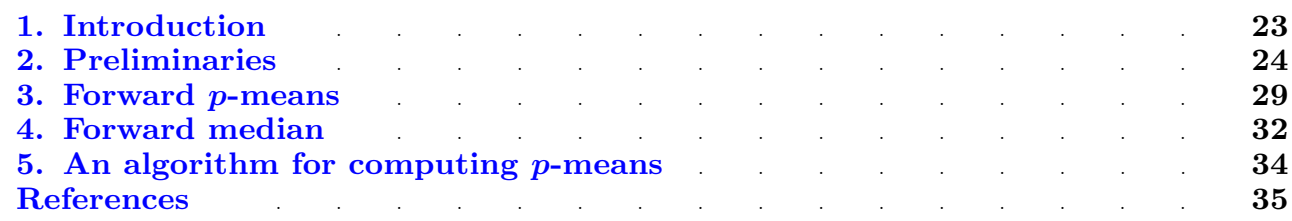

\section{Introduction}

The geometric barycenter of a set of points is the point which minimizes the sum of the squared distances to these points. It is the most traditional estimator in statistics that is however sensitive to outliers $[\mathbf{1 8}]$. Thus, it is natural to replace the average distance squaring (power 2) by taking the power of $p$ for some $p \in[1,2)$. This leads to the definition of $p$-means. When $p=1$, the minimizer is the median of the set of points, very often used in robust statistics [18]. In many applications, $p$-means with some $p \in(1,2)$ give the best compromise. For existence and uniqueness in Riemannian manifolds under convexity conditions on the support of the measure, see Afsari [1].

The Fermat-Weber problem concerns finding the median $e_{1}$ of a set of points in a Euclidean space. Numerous authors worked out algorithms for computing $e_{1}$. The first algorithm was proposed by Weiszfeld in [34] (see also [33]). It has been extended to sufficiently small domains in Riemannian manifolds with non-negative curvature by Fletcher et al. in [13]. A complete generalization to manifolds with positive or negative curvature (under some convexity conditions in positive curvature) has been recently given by Yang in [36].

The Riemannian barycenter or Karcher mean of a set of points in a manifold or more generally of a probability measure has been extensively studied; see for example $[4,9,12,19-$ 21, 30], where questions of existence, uniqueness, stability, relation with martingales in manifolds, and behavior when measures are pushed by stochastic flows have been considered. The Riemannian barycenter corresponds to $p=2$ in the above description. Computation of Riemannian barycenters by gradient descent has been performed by Le in [23].

The aim of this paper is to extend to the context of Finsler manifolds the results on existence and uniqueness of $p$-means of probability measures, as well as algorithms for computing them.

Received 26 November 2010; revised 11 September 2011.

2000 Mathematics Subject Classification 53B40 (primary). 
Some convexity is needed, and as we shall see the fact that comparison results for triangles as Alexandroff and Toponogov theorems do not exist imposes more restrictions on the support of the probability measure. As a consequence, the sharp results on existence and uniqueness established by Afsari [1] and the algorithm for computing means of Yang in [36] do not extend to Finsler manifolds.

The motivation for this work primarily comes from signal filtering and denoising in the context of diffusion tensor imaging (DTI), high angular resolution diffusion imaging (HARDI, see [5, 28, 32]), orientation distribution functions (ODFs), and active contours [24]. Applications with experimental results of an implementation will be reported in forthcoming papers.

Information geometry at its heart considers the differential geometry nature of probability distributions induced by a divergence function. In probability theory, invariance by monotonic re-parameterization and sufficient statistics yields the class of $f$-divergences [3] $I_{f}(p, q)=\int p(x) f(q(x) / p(x)) d x$ that includes the Kullback-Leibler (KL) information-theoretic divergence $\mathrm{KL}(p, q)=\int p(x) \log (p(x) / q(x)) d x$ as its prominent member (for $f(t)=-\log t$ ). It is well known that the KL divergence (better known as the relative entropy) yields a dually flat structure $[\mathbf{3}]$ generalizing the (self-dual) Euclidean space.

Because divergences are usually asymmetric and violate the triangle inequality, they have not been extensively considered from an algorithmic point of view. Indeed, the triangle inequality property is often used in computational geometry to design efficient algorithms by allowing various 'pruning' techniques $[\mathbf{1 1}, \mathbf{1 5}]$. Computational geometry has thus mostly considered metric spaces for keeping the triangle inequality properties.

One can metrize divergences. The KL divergence can be symmetrized either into the Jeffreys divergence $J(p, q)=\mathrm{KL}(p, q)+\mathrm{KL}(q, p)$ or into the Jensen-Shannon (JS) divergence:

$$
\begin{aligned}
\operatorname{JS}(p, q) & =\mathrm{KL}\left(p, \frac{p+q}{2}\right)+\mathrm{KL}\left(q, \frac{p+q}{2}\right) \\
& =\int\left(p(x) \log \frac{2 p(x)}{p(x)+q(x)}+q(x) \log \frac{2 q(x)}{p(x)+q(x)}\right) d x .
\end{aligned}
$$

The latter is preferred in practice because it is bounded, and its square root yields a metric that can be embedded into a Hilbert space [14].

Finsler distances, arising from the underlying Finsler metrics, are attractive as they preserve the triangle inequality [32] for efficient algorithmics but potentially model asymmetric distances.

In information geometry, the regular divergence $D$ associated to a Finslerian metric distance $d$ can be defined as $D(p, q)=d^{2}(p, q)$. Observe that the Finslerian-based divergence loses then the triangle inequality property [32] (for example, the squared Euclidean distance does not satisfy the triangle inequality).

\section{Preliminaries}

Let $M$ be a smooth manifold. On $M$ we consider a Finsler structure $F: T M \rightarrow \mathbb{R}_{+}$. For any $x \in M, V, X, Y, Z \in T_{x} M$ such that $V \neq 0$, let

$$
g_{V}(X, Y):=\left.\frac{1}{2} \frac{\partial^{2}}{\partial s \partial t}\right|_{(s, t)=(0,0)} F^{2}(V+s X+t Y)
$$

(we shall also use the notation $\langle X, Y\rangle_{V}=g_{V}(X, Y)$ ) and

$$
\langle X, Y, Z\rangle_{V}:=\left.\frac{1}{4} \frac{\partial^{3}}{\partial r \partial s \partial t}\right|_{(r, s, t)=(0,0,0)} F^{2}(V+r X+s Y+t Z) .
$$


We have

$$
\langle X, Y, Z\rangle_{V}=\left.\frac{1}{2} \frac{\partial}{\partial r}\right|_{r=0} g_{V+r X}(Y, Z)
$$

and in particular since $F^{2}$ is 2-homogeneous and $V \mapsto g_{V}(X, Y)$ is 0-homogeneous,

$$
\langle V, Y, Z\rangle_{V}=0 .
$$

Let $V$ be a non-vanishing vector field on $M$. The Chern connection $\nabla^{V}$ is torsionfree and almost metric, and can be characterized by

$$
X\langle Y, Z\rangle_{V}=\left\langle\nabla_{X}^{V} Y, Z\right\rangle_{V}+\left\langle Y, \nabla_{X}^{V} Z\right\rangle_{V}+2\left\langle\nabla_{X}^{V} V, Y, Z\right\rangle_{V}
$$

More precisely, parameterizing locally $T M$ by coordinates

$$
\left(x^{1}, \ldots, x^{m}, y^{1}=d x^{1}, \ldots, y^{m}=d x^{m}\right),
$$

defining the geodesic coefficients as

$$
G^{i}(y)=\frac{1}{4} g^{i k}(y)\left(2 \frac{\partial g_{j k}}{\partial x^{l}}-\frac{\partial g_{j l}}{\partial x^{k}}\right) y^{j} y^{l}, \quad y \in T M \backslash\{0\},
$$

and letting

$$
N_{j}^{i}=\frac{\partial G^{i}}{\partial y^{j}}, \quad \frac{\delta}{\delta x^{i}}=\frac{\partial}{\partial x^{i}}-N_{i}^{k}(y) \frac{\partial}{\partial y^{k}} \in T_{y}(T M \backslash\{0\}),
$$

the Christoffel symbols of the Chern connection are given by

$$
\Gamma_{i j}^{k}=\frac{1}{2} g^{k l}\left(\frac{\delta g_{l j}}{\delta x^{i}}+\frac{\delta g_{i l}}{\delta x^{j}}-\frac{\delta g_{i j}}{\delta x^{l}}\right)
$$

(see $[8])$. Note that defining

$$
\delta y^{i}=d y^{i}+N_{j}^{i}(y) d x^{j},
$$

we have for a smooth function $f: T M \backslash\{0\} \rightarrow \mathbb{R}$

$$
d f=\frac{\delta f}{\delta x^{i}} d x^{i}+\frac{\partial f}{\partial y^{i}} \delta y^{i} .
$$

The Chern curvature tensor is defined by the equation

$$
R^{V}(X, Y) Z:=\nabla_{X}^{V} \nabla_{Y}^{V} Z-\nabla_{Y}^{V} \nabla_{X}^{V} Z-\nabla_{[X, Y]}^{V} Z,
$$

and the flag curvature is

$$
\mathscr{K}(V, W):=\frac{\left\langle R^{V}(V, W) W, V\right\rangle}{\langle V, V\rangle_{V}\langle W, W\rangle_{V}-\langle V, W\rangle_{V}^{2}},
$$

for two non-collinear $V, W \in T_{x} M$.

We say that $M$ has non-positive flag curvature if, for all $V, W, \mathscr{K}(V, W) \leq 0$.

The tangent curvature of two vectors $V, W \in T_{x} M$ is defined as

$$
\mathscr{T}_{V}(W)=\left\langle\nabla_{W}^{W} \tilde{W}-\nabla_{W}^{V} \tilde{W}, V\right\rangle_{V}
$$

where $\tilde{W}$ is a vector field satisfying $\tilde{W}_{x}=W$. For a non-negative constant $\delta \geq 0$, we say that $\mathscr{T} \geq-\delta$ or $\mathscr{T} \leq \delta$ if, respectively,

$$
\mathscr{T}_{V}(W) \geq-\delta F(V) F(W)^{2} \quad \text { or } \mathscr{T}_{V}(W) \leq \delta F(V) F(W)^{2} .
$$

For $x \in M$, we define

$$
\mathscr{C}(x)=\sup _{v, w \in T_{x} M \backslash\{0\}} \sqrt{\frac{\langle v, v\rangle_{v}}{\langle v, v\rangle_{w}}}, \quad \mathscr{D}(x)=\sup _{v, w \in T_{x} M \backslash\{0\}} \sqrt{\frac{\langle v, v\rangle_{w}}{\langle v, v\rangle_{v}}} .
$$


REMARK 2.1. For applications in active contours, a 'Wulff shape' is given which does not depend on $x$ and defines the Finsler structure. From this shape, $\mathscr{C}$ and $\mathscr{D}$ can easily be calculated. See for example $[\mathbf{2 4}, \mathbf{3 7}]$.

A geodesic in $M$ is a curve $t \mapsto c(t)$ satisfying for all $t, \nabla_{\dot{c}(t)}^{\dot{c}(t)} \dot{c}=0$. It is well known that a geodesic has constant speed, and that it locally minimizes the distance [8]. If so, letting $\rho(x, y)$ be the forward distance from $x$ to $y$,

$$
\rho^{2}(x, y)=\langle\dot{c}(0), \dot{c}(0)\rangle_{\dot{c}(0)},
$$

where $t \mapsto c(t)$ is the minimal geodesic satisfying $c(0)=x$ and $c(1)=y$. By definition, the backward distance from $x$ to $y$ is $\rho(y, x)$.

For $x \in M$ and $v \in T_{x} M$, we let whenever it exists $\exp _{x}(v):=c(1)$, where $t \mapsto c(t)$ is the geodesic satisfying $\dot{c}(0)=v$.

If $M$ is complete, analytic, simply connected, and has non-positive flag curvature (we say that $M$ is an analytic Cartan-Hadamard manifold), then $\exp _{x}: T_{x} M \rightarrow M$ is a homeomorphism [6, Theorem 4.7]. Under these assumptions, letting for $x, y \in M, \overrightarrow{x y}=\exp _{x}^{-1}(y)$, we have

$$
\rho^{2}(x, y)=\langle\overrightarrow{x y}, \overrightarrow{x y}\rangle_{\overrightarrow{x y}} .
$$

For $x_{0} \in M$ and $R>0$, let us denote by $B\left(x_{0}, R\right)$ (respectively $\left.\bar{B}\left(x_{0}, R\right)\right)$ the (forward) open (respectively closed) ball with center $x_{0}$ and radius $R$ :

$$
B\left(x_{0}, R\right)=\left\{y \in M, \rho\left(x_{0}, y\right)<R\right\} \quad \text { (respectively } \bar{B}\left(x_{0}, R\right)=\left\{y \in M, \rho\left(x_{0}, y\right) \leq R\right\} \text { ) }
$$

Now let $(t, s) \mapsto c(t, s)$ be a family of minimizing geodesics $t \mapsto c(t, s), \quad t \in[0,1]$, parameterized by $s \in I, I$ being an interval in $\mathbb{R}$. Define

$$
E(s)=\frac{1}{2} \rho^{2}(c(0, s), c(1, s)) .
$$

The computation of $E^{\prime}(s)$ and $E^{\prime \prime}(s)$ is well known; see for example [7]. We have

$$
E^{\prime}(s)=\left\langle\partial_{s} c(1, s), \partial_{t} c(1, s)\right\rangle_{\partial_{t} c(1, s)}-\left\langle\partial_{s} c(0, s), \partial_{t} c(0, s)\right\rangle_{\partial_{t} c(0, s)} .
$$

As for the second derivative, letting $c=c(\cdot, 0)$ and, for $X, Y$ vector fields along $c$,

$$
I(X, Y)=\int_{0}^{1}\left(\left\langle\nabla_{T}^{T} X, \nabla_{T}^{T} Y\right\rangle_{T}-\left\langle R^{T}(X, T) T, Y\right\rangle_{T}\right) d t
$$

be the index of $X$ and $Y$, we have

$$
\begin{aligned}
E^{\prime \prime}(0)= & \left\langle\nabla_{\partial_{s} c(1,0)}^{\partial_{t} c(1,0)} \partial_{s} c(1, \cdot), \partial_{t} c(1,0)\right\rangle_{\partial_{t} c(1,0)}-\left\langle\nabla_{\partial_{s} c(0,0)}^{\partial_{t} c(0,0)} \partial_{s} c(0, \cdot), \partial_{t} c(0,0)\right\rangle_{\partial_{t} c(0,0)} \\
& +I\left(\partial_{s} c(\cdot, 0), \partial_{s} c(\cdot, 0)\right) .
\end{aligned}
$$

Assuming $s \mapsto c(0, s)$ and $s \mapsto c(1, s)$ are geodesics, we obtain

$$
E^{\prime \prime}(0)=\mathscr{T}_{\partial_{t} c(0,0)}\left(\partial_{s} c(0,0)\right)-\mathscr{T}_{\partial_{t} c(1,0)}\left(\partial_{s} c(1,0)\right)+I\left(\partial_{s} c(\cdot, 0), \partial_{s} c(\cdot, 0)\right) .
$$

We are interested in the situation where $c(1, s) \equiv z$ a constant. In this case, we have

$$
E^{\prime \prime}(0)=\mathscr{T}_{\partial_{t} c(0,0)}\left(\partial_{s} c(0,0)\right)+I\left(\partial_{s} c(\cdot, 0), \partial_{s} c(\cdot, 0)\right) .
$$

For $p \geq 1$, define

$$
D_{p}(s)=\rho^{p}(c(0, s), z)
$$

Proposition 2.2. Assume $\mathscr{K} \leq k, \mathscr{T} \geq-\delta$, and $\mathscr{C} \leq C$ for some $k, \delta \geq 0, C \geq 1$. Let $p>1$. Then, writing $r=\rho(x, z)$,

$$
D_{p}^{\prime \prime}(0) \geq p r^{p-2}\left(\min \left(p-1, \frac{\sqrt{k} r \cos (\sqrt{k} r)}{\sin (\sqrt{k} r)}\right) C^{-2}-\delta r\right) .
$$


If $z$ and $x=c(0,0)$ satisfy $\rho(x, z)<R(p, k, \delta, C)$ with

$$
R(p, k, \delta, C)=\min \left(\frac{p-1}{C^{2} \delta}, \frac{1}{\sqrt{k}} \arctan \left(\frac{\sqrt{k}}{C^{2} \delta}\right)\right)
$$

and the injectivity radius at $x$ is strictly larger than $R(p, k, \delta, C)$, then $D_{p}^{\prime \prime}(0)>0$.

REMARK 2.3. Note if $p \geq 2$, then

$$
R(p, k, \delta, C)=R(2, k, \delta, C)=\frac{1}{\sqrt{k}} \arctan \left(\frac{\sqrt{k}}{C^{2} \delta}\right) .
$$

Proof. Define $T(t)=\partial_{t} c(t, 0), J(t)=\partial_{s} c(t, 0)$, and

$$
J^{T}(t)=\frac{1}{F(T(t))^{2}}\langle J(t), T(t)\rangle_{T(t)} T(t), \quad J^{N}(t)=J(t)-J^{T}(t) .
$$

Using successively [7, Lemma 9.5.1] which compares the index $I(J, J)$ with the one of its 'transplant' into a manifold with constant curvature $k^{2}$ and the index lemma [7, Lemma 7.3.2] which compares the index of the transplant to the one of the Jacobi field with the same boundary values, we get, letting $r=\rho(x, z)=D_{1}(0)$,

$$
I(J, J) \geq \frac{\sqrt{k} r \cos (\sqrt{k} r)}{\sin (\sqrt{k} r)}\left\langle J^{N}(0), J^{N}(0)\right\rangle_{T(0)}+\left\langle J^{T}(0), J^{T}(0)\right\rangle_{T(0)} .
$$

Using the expression (2.24) for $E^{\prime \prime}(0)$, we obtain

$$
E^{\prime \prime}(0) \geq-\delta r+\frac{\sqrt{k} r \cos (\sqrt{k} r)}{\sin (\sqrt{k} r)}\left\langle J^{N}(0), J^{N}(0)\right\rangle_{T(0)}+\left\langle J^{T}(0), J^{T}(0)\right\rangle_{T(0)} .
$$

We have from (2.20)

$$
E^{\prime}(0)^{2}=r^{2}\left\langle J^{T}(0), J^{T}(0)\right\rangle_{T(0)} .
$$

Now, from $D_{1}(s)=\sqrt{2 E(s)}$, we get

$$
D_{1}^{\prime}(s)=\frac{E^{\prime}(s)}{D_{1}(s)}, \quad D_{1}^{\prime \prime}(s)=\frac{E^{\prime \prime}(s)}{D_{1}(s)}-\frac{E^{\prime}(s)^{2}}{D_{1}^{3}(s)}
$$

and this yields

$$
\begin{aligned}
D_{p}^{\prime \prime}(0) & =p D_{1}(0)^{p-2}\left((p-1) D_{1}^{\prime}(0)^{2}+D_{1}(0) D_{1}^{\prime \prime}(0)\right) \\
& =p r^{p-2}\left((p-2)\left\langle J^{T}(0), J^{T}(0)\right\rangle_{T(0)}+E^{\prime \prime}(0)\right) \\
& \geq p r^{p-2}\left((p-1)\left\langle J^{T}(0), J^{T}(0)\right\rangle_{T(0)}-\delta r+\frac{\sqrt{k} r \cos (\sqrt{k} r)}{\sin (\sqrt{k} r)}\left\langle J^{N}(0), J^{N}(0)\right\rangle_{T(0)}\right) \\
& \geq p r^{p-2}\left(\min \left(p-1, \frac{\sqrt{k} r \cos (\sqrt{k} r)}{\sin (\sqrt{k} r)}\right)\langle J(0), J(0)\rangle_{T(0)}-\delta r\right) \\
& \geq p r^{p-2}\left(\min \left(p-1, \frac{\sqrt{k} r \cos (\sqrt{k} r)}{\sin (\sqrt{k} r)}\right) C^{-2}-\delta r\right) .
\end{aligned}
$$

From this bound the rest of the proof follows easily.

Similarly, we can obtain an upper bound for $D_{p}^{\prime \prime}(0)$.

Proposition 2.4. Assume the sectional curvatures $\mathscr{K}$ have a lower bound $-\beta^{2}$ for some $\beta>0$, and $\mathscr{T} \leq \delta^{\prime}$ for some $\delta^{\prime}>0, \mathscr{D} \leq D$ for some $D \geq 1$. Again, let $r=\rho(x, z)$ and assume 
that the injectivity radius at $x$ is larger than $r$. Then

$$
D_{p}^{\prime \prime}(0) \leq p r^{p-2}\left(D^{2} \max (p-1, \beta r \operatorname{coth}(\beta r))+\delta^{\prime} r\right) .
$$

Proof. We have by (2.24) and (2.31) together with the fact that

$$
\begin{gathered}
I(J, J)=\left\langle J^{T}(0), J^{T}(0)\right\rangle+I\left(J^{N}, J^{N}\right), \\
\left.D_{p}^{\prime \prime}(0)=p r^{p-2}(p-1)\left\langle J^{T}(0), J^{T}(0)\right\rangle_{T(0)}+I\left(J^{N}, J^{N}\right)+\mathscr{T}_{T}(J)\right) .
\end{gathered}
$$

Let $t \mapsto X(t)$ be the parallel vector field along $t \mapsto c(t, 0)$ with initial condition $J^{N}(0)$ and, for $t \in[0,1]$, let

$$
G(t)=\cosh (r \beta t)-\operatorname{coth}(r \beta) \sinh (r \beta t) .
$$

This is the solution of $G^{\prime \prime}=r \beta G$ with conditions $G(0)=1$ and $G(1)=0$. The vector field $t \mapsto Y(t)$ along $t \mapsto c(t, 0)$ defined by

$$
Y(t)=G(t) X(t)
$$

has the same boundary values as $t \mapsto J^{N}(t)$, so, by the index lemma [7, Lemma 7.3.2], we have

$$
I\left(J^{N}, J^{N}\right) \leq I(Y, Y)
$$

On the other hand,

$$
\begin{aligned}
I(Y, Y) & =\int_{0}^{1}\left(G^{\prime}(t)^{2}\left\langle J^{N}(0), J^{N}(0)\right\rangle_{T(0)}-G(t)^{2}\left\langle R^{T}(X(t), T(t)) T(t), X(t)\right\rangle_{T(t)}\right) d t \\
& \leq\left\langle J^{N}(0), J^{N}(0)\right\rangle_{T(0)} \int_{0}^{1}\left(G^{\prime}(t)^{2}+r^{2} \beta^{2} G(t)^{2}\right) d t \\
& =\left\langle J^{N}(0), J^{N}(0)\right\rangle_{T(0)}\left(\left[G^{\prime}(t) G(t)\right]_{0}^{1}+\int_{0}^{1} G(t)\left(-G^{\prime \prime}(t)^{2}+r^{2} \beta^{2} G(t)\right) d t\right) \\
& =\left\langle J^{N}(0), J^{N}(0)\right\rangle_{T(0)} r \beta \operatorname{coth}(r \beta) .
\end{aligned}
$$

So,

$$
\begin{aligned}
D_{p}^{\prime \prime}(0) & \leq p r^{p-2}\left((p-1)\left\langle J^{T}(0), J^{T}(0)\right\rangle_{T(0)}+r \beta \operatorname{coth}(r \beta)\left\langle J^{N}(0), J^{N}(0)\right\rangle_{T(0)}\right)+\delta^{\prime} r \\
& \leq p r^{p-2}\left(\max ((p-1), r \beta \operatorname{coth}(r \beta))\langle J(0), J(0)\rangle_{T(0)}+\delta^{\prime} r\right) \\
& \leq p r^{p-2}\left(D^{2} \max ((p-1), r \beta \operatorname{coth}(r \beta))+\delta^{\prime} r\right),
\end{aligned}
$$

since $F(J(0))=1$.

For $x \in M$, let $\ell_{x}: T_{x} M \rightarrow T_{x}^{*} M$ be the Legendre transformation, defined as

$$
\ell_{x}(V)=g_{V}(V, \cdot) \quad \text { if } V \neq 0, \quad \ell_{x}(0)=0 .
$$

It is well known that $\ell_{x}$ is a bijection. The global Legendre transformation on $T M$ is defined as

$$
\mathscr{L}(V)=\ell_{\pi(V)}(V),
$$

where $\pi: T M \rightarrow M$ is the canonical projection. If we define the dual Minkowski norm $F^{*}$ on $T_{x}^{*} M$ as

$$
F^{*}(\xi)=\max \left\{\xi(y), y \in T_{x} M, F(y)=1\right\},
$$

then

$$
F=F^{*} \circ \mathscr{L}
$$


and, for non-zero $V \in T M$ and $\alpha \in T^{*} M$,

$$
\langle\mathscr{L}(V), V\rangle=F(V)^{2}, \quad\left\langle\alpha, \mathscr{L}^{-1}(\alpha)\right\rangle=F^{*}(\alpha)^{2}
$$

(see for example [2]).

For $f$ a $C^{1}$ function on $M$, we may define the gradient of $f$ as

$$
\operatorname{grad} f=\mathscr{L}^{-1}(d f) \text {. }
$$

\section{Forward p-means}

Let $\mu$ be a compactly supported probability measure in $M$. For $p>1$ and $x \in M$, we define

$$
\mathscr{E}_{\mu, p}(x)=\int_{M} \rho^{p}(x, z) \mu(d z) .
$$

The (forward) $p$-mean of $\mu$ is the point $e_{p}$ of $M$ where $\mathscr{E}_{\mu, p}$ reaches its minimum whenever it exists and is unique.

In this paper we will consider forward $p$-means and we will call them $p$-means. Similarly, we could define the backward $p$-mean $\overleftarrow{e}_{p}$ as the point which minimizes

$$
x \mapsto \overleftarrow{\mathscr{E}}_{\mu, p}(x):=\int_{M} \rho^{p}(z, x) \mu(d z)
$$

Depending on the context, forward or backward mean is more appropriate. One should note that defining the reverse (or adjoint) Finsler structure $\overleftarrow{F}(v)=F(-v), v \in T M$, it is easy to check that the associated distance $\overleftarrow{\rho}$ satisfies $\overleftarrow{\rho}(z, x)=\rho(x, z)$, and the forward $p$-mean for $\overleftarrow{F}$ is the backward $p$-mean for $F$. So, without loss of generality, we can consider only the forward $p$-means.

One should also note that in high angular resolution diffusion imaging the Finsler structure is symmetric, so both notions coincide. It is not the case for the application concerning active contours, where it is natural to consider non-symmetric $F$.

Even if it is in a non-Finslerian context, one can give the example of right-sided and leftsided Kullback-Leibler divergences for families of Gaussian probability densities (see [25]). The left-sided centroid focuses on the highest mode (it is zero-forcing), and the rightsided centroid tries to cover the support of both normals (it is zero-avoiding as depicted in [25, Figure 2]). Furthermore, the left-sided Kullback-Leibler centroid yields the best single Gaussian distribution approximating the mixture model induced by the (weighted) family of Gaussians [29, Theorem 4.1]. The left-sided Kullback-Leibler centroid has been successfully used for simplifying statistical mixture models [17].

Proposition 3.1. Assume there exists $C>0$ such that $\mathscr{C}(x) \leq C$ for all $x \in M$, where $\mathscr{C}(x)$ is defined in (2.15). Assume furthermore that $\operatorname{supp}(\mu) \subset B\left(x_{0}, R\right)$ for some $x_{0} \in M$ and $R>0$. Then $x \mapsto \mathscr{E}_{\mu, p}(x)$ has at least one global minimum in $\bar{B}\left(x_{0}, C(1+C) R\right)$.

Proof. We begin with establishing that for all $y_{1}, y_{2} \in M$,

$$
\frac{1}{C} \rho\left(y_{2}, y_{1}\right) \leq \rho\left(y_{1}, y_{2}\right) \leq C \rho\left(y_{2}, y_{1}\right) .
$$

It is sufficient to establish the second inequality and then to exchange $y_{1}$ and $y_{2}$. If $t \mapsto \varphi(t)$ is a path from $y_{1}=\varphi(0)$ to $y_{2}=\varphi(1)$, then its length $L(\varphi)$ satisfies

$$
\begin{aligned}
L(\varphi) & =\int_{0}^{1} \sqrt{\langle\dot{\varphi}(t), \dot{\varphi}(t)\rangle_{\dot{\varphi}(t)}} d t \\
& =\int_{0}^{1} \sqrt{\frac{\langle-\dot{\varphi}(t),-\dot{\varphi}(t)\rangle_{\dot{\varphi}(t)}}{\langle-\dot{\varphi}(t),-\dot{\varphi}(t)\rangle_{-\dot{\varphi}(t)}}} \sqrt{\langle-\dot{\varphi}(t),-\dot{\varphi}(t)\rangle_{-\dot{\varphi}(t)}} d t
\end{aligned}
$$




$$
\begin{aligned}
& \leq \int_{0}^{1} \mathscr{C}(\varphi(t)) \sqrt{\langle-\dot{\varphi}(t),-\dot{\varphi}(t)\rangle_{-\dot{\varphi}(t)}} d t \\
& \leq C \int_{0}^{1} \sqrt{\langle-\dot{\varphi}(t),-\dot{\varphi}(t)\rangle_{-\dot{\varphi}(t)}} d t \\
& =C L(\hat{\varphi}),
\end{aligned}
$$

where $\hat{\varphi}$ is the path from $y_{2}$ to $y_{1}$ defined by $\hat{\varphi}(t)=\varphi(1-t)$. Minimizing over all paths $\hat{\varphi}$ from $y_{2}$ to $y_{1}$, we get

$$
\rho\left(y_{1}, y_{2}\right) \leq C \rho\left(y_{2}, y_{1}\right) .
$$

Now, if $\operatorname{supp}(\mu) \subset B\left(x_{0}, R\right)$, then $\mathscr{E}_{\mu, p}\left(x_{0}\right) \leq R^{p}$. On the other hand, if $x \notin \bar{B}\left(x_{0}\right.$, $C(1+C) R)$, then, for all $y \in B\left(x_{0}, R\right)$,

$$
\begin{aligned}
\rho(x, y) & \geq \rho\left(x, x_{0}\right)-\rho\left(y, x_{0}\right) \\
& \geq \frac{1}{C} \rho\left(x_{0}, x\right)-C \rho\left(x_{0}, y\right) \\
& \geq(1+C) R-C R=R
\end{aligned}
$$

and this clearly implies that $\mathscr{E}_{\mu, p}(x) \geq R^{p}$. From this, we get the conclusion.

Concerning the uniqueness of the global minimum of $\mathscr{E}_{\mu, p}$, we also have the following easy result.

Proposition 3.2. Assume that $\mu$ is supported by a compact ball $\bar{B}\left(x_{0}, R\right)$ and that, for all $z \in \bar{B}\left(x_{0}, R\right)$, the function $x \mapsto \rho^{p}(x, z)$ is strictly convex in $\bar{B}\left(x_{0}, C(1+C) R\right)$. Then $\mu$ has a unique $p$-mean in $\bar{B}\left(x_{0}, C(1+C) R\right)$.

Proof. If $x \mapsto \rho^{p}(x, z)$ is strictly convex for all $z$ in the support of $\mu$, then $\mathscr{E}_{\mu, p}$ is strictly convex, and this implies that it has a unique minimum, which is attained at a unique point $e_{p}$. $\square$

Corollary 3.3. Assume $\mathscr{K} \leq k, \mathscr{T} \geq-\delta$, and $\mathscr{C} \leq C$ for some $k, \delta \geq 0, C \geq 1$. Let $p>1$. Again, let

$$
R(p, k, \delta, C)=\min \left(\frac{p-1}{C^{2} \delta}, \frac{1}{\sqrt{k}} \arctan \left(\frac{\sqrt{k}}{C^{2} \delta}\right)\right) .
$$

If $\mu$ is supported by a geodesic ball $B\left(x_{0}, R\right)$ with

$$
R \leq \frac{1}{C(C+1)^{2}} R(p, k, \delta, C)
$$

and the injectivity radius at any $x \in B\left(x_{0}, C(1+C) R\right)$ is strictly larger than $R(p, k, \delta, C)$, then $\mu$ has a unique $p$-mean $e_{p}$ satisfying

$$
e_{p} \in \bar{B}\left(x_{0}, \frac{1}{C+1} R(p, k, \delta, C)\right) .
$$

Proof. If $x, z \in B\left(x_{0}, C(1+C) R\right)$, then

$$
\rho(x, z) \leq \rho\left(x, x_{0}\right)+\rho\left(x_{0}, z\right) \leq(1+C)^{2} C R \leq R(p, k, \delta, C) .
$$

Using Proposition 2.2, we obtain that $\mathscr{E}_{\mu, p}$ is strictly convex on $B\left(x_{0}, C(1+C) R\right)$. So, by Proposition $3.2, \mu$ has a unique $p$-mean in $\bar{B}\left(x_{0}, C(1+C) R\right)$.

REMARK 3.4. Letting $x_{0} \in M$ and $D$ be a relatively compact neighborhood of $x_{0}$, then $\mathscr{K}$ and $\mathscr{C}$ are bounded above on $D$ by, say, $k_{D}$ and $C_{D}$, and $\mathscr{T}$ is bounded below on $D$ by $-\delta_{D}$. 
Using these bounds instead of $k, C$, and $\delta$, we can find $R$ sufficiently small so that the conditions of Corollary 3.3 are fulfilled. So, we can say any measure $\mu$ with sufficiently small support has a unique $p$-mean.

Remark 3.5. If $M$ is a Cartan-Hadamard manifold, we recover the fact that we can take $R(p, k, \delta, C)$ as large as we want.

More generally, in the Riemannian case, Afsari [1] proved existence and uniqueness of $p$-means, $p \geq 1$, on geodesic balls with radius

$$
r<\frac{1}{2} \min \left\{\operatorname{inj}(M), \frac{\pi}{2 \sqrt{k}}\right\} \quad \text { if } p \in[1,2)
$$

and

$$
r<\frac{1}{2} \min \left\{\operatorname{inj}(M), \frac{\pi}{\sqrt{k}}\right\} \quad \text { if } p \geq 2 .
$$

Even taking $\delta=0$ and $C=1$ in Corollary 3.3, the support of $\mu$ has half the size of the one in [1] for $p \in(1,2)$, due to the fact that we have an additional condition (3.4) coming from the non-optimality of Proposition 3.2 in the Riemannian context. As for $p \geq 2$, another factor of two is gained in $[\mathbf{1}]$ with repeated use of Toponogov and Alexandroff theorems which are not available in our context.

REMARK 3.6. The condition on injectivity radius is the same as in the Riemannian case. The cut locus of any point of $x \in B\left(x_{0}, C(1+C) R\right)$ has to be at distance larger than $R(p, k, \delta, C)$. As for Riemannian manifolds, there is no general condition which insures this property for cut points, but for conjugate points the same condition holds, due to the Rauch comparison theorem; see [7, Theorem 9.6.1]. In the particular case when $M$ is a CartanHadamard Finsler manifold, that is, it has non-positive flag curvature and it is simply connected, the injectivity radius in everywhere infinite (see [7, Theorem 9.4.1]).

Proposition 3.7. Let $a \mapsto x(a)$ solve the equation

$$
x(0)=x_{0} \quad \text { and for } a \geq 0 \quad x^{\prime}(a)=\operatorname{grad}_{x(a)}\left(-\mathscr{E}_{\mu, p}\right) .
$$

Under the conditions of Corollary 3.3, the path $a \mapsto x(a)$ converges as $a \rightarrow \infty$ to the p-mean of $\mu$.

Proof. If $f(a)=\left(-\mathscr{E}_{\mu}\right)(x(a))$, we have, as soon as $\operatorname{grad}_{x(a)}\left(-\mathscr{E}_{\mu, p}\right) \neq 0$,

$$
\begin{aligned}
f^{\prime}(a) & =\left\langle d_{x(a)}\left(-\mathscr{E}_{\mu, p}\right), x^{\prime}(a)\right\rangle \\
& =\left\langle d_{x(a)}\left(-\mathscr{E}_{\mu, p}\right), \operatorname{grad}_{x(a)}\left(-\mathscr{E}_{\mu, p}\right)\right\rangle \\
& =\left\langle d_{x(a)}\left(-\mathscr{E}_{\mu, p}\right), \mathscr{L}^{-1}\left(d_{x(a)}\left(-\mathscr{E}_{\mu, p}\right)\right)\right\rangle \\
& =F^{*}\left(d_{x(a)}\left(-\mathscr{E}_{\mu, p}\right)\right)^{2}
\end{aligned}
$$

by $(2.40)$ and (2.41).

On the other hand, we have $f(0) \geq-R^{p}$ and $f$ is non-decreasing. This implies that for all $a \geq 0, x(a) \in \bar{B}\left(x_{0}, C(1+C) R\right)$, since, for all $x \notin \bar{B}\left(x_{0}, C(1+C) R\right), \mathscr{E}_{\mu, p}(x) \geq R^{p}$. As a consequence, $x(a)$ has limit points as $a$ goes to infinity and, since $f(a)$ converges, any limit point is a critical point of $x \mapsto \mathscr{E}_{\mu, p}(x)$. But, by Proposition $3.2, \mathscr{E}_{\mu, p}$ has a unique critical point in $\bar{B}\left(x_{0}, C(1+C) R\right)$ which is the mean $e_{p}$ of $\mu$. So, we can conclude that $x(a)$ converges to $e_{p}$. 


\section{Forward median}

Let $\mu$ be a compactly supported probability measure in $M$. For $x \in M$, we define

$$
\mathscr{F}_{\mu}(x)=\int_{M} \rho(x, z) \mu(d z) .
$$

The median of $\mu$ is the point in $M$ where $\mathscr{F}_{\mu}$ reaches its minimum whenever it exists and is unique.

In the terminology of Section 3, we have $\mathscr{F}_{\mu}=\mathscr{E}_{\mu, 1}$. The following result extends Proposition 3.1 to the case $p=1$ and has exactly the same proof.

Proposition 4.1. Assume there exists $C>0$ such that $\mathscr{C}(x) \leq C$ for all $x \in M$. Assume furthermore that $\operatorname{supp}(\mu) \subset B\left(x_{0}, R\right)$ for some $x_{0} \in M$ and $R>0$. Then $x \mapsto \mathscr{F}_{\mu}(x)$ has at least one global minimum in $\bar{B}\left(x_{0}, C(1+C) R\right)$.

Proposition 4.2. Assume that $\mu$ is supported by a compact ball $\bar{B}\left(x_{0}, R\right)$, that the support of $\mu$ is not contained in a single geodesic, and that, for all $z \in \bar{B}\left(x_{0}, R\right)$, the forward distance to $z$ is convex, and strictly convex in any geodesic of $\bar{B}\left(x_{0}, C(1+C) R\right)$ which does not contain $z$. Then $\mu$ has a unique median $m \in \bar{B}\left(x_{0}, C(1+C) R\right)$.

Proof. Clearly, under these assumptions $\mathscr{F}_{\mu}$ is strictly convex, so it has a unique local minimum; this minimum is global and is attained at a unique point $m \in \bar{B}\left(x_{0}, C(1+C) R\right)$.

REMARK 4.3. Contrarily to the case of $p$-means for $p>1$, we cannot say at this stage that any probability measure $\mu$ with sufficiently small support has a unique median, since we do not know whether $\mathscr{F}_{\mu}$ is strictly convex or not. In the next proposition, we give a sufficient condition for strict convexity of $\mathscr{F}_{\mu}$.

Proposition 4.4. Assume $\mathscr{K} \leq k$ and $\mathscr{T} \geq-\delta$ for some $k, \delta>0$. Assume that the injectivity radius at any point of $\bar{B}\left(x_{0}, C(1+C) R\right)$ is larger than $\left(C^{2}+C+1\right) R$. Define

$$
\begin{aligned}
\eta= & \min \left\{\int_{M} \sqrt{k} \cot (\sqrt{k} \rho(\pi(v), z))\left\langle v^{N}, v^{N}\right\rangle \overrightarrow{\pi(v) z} \mu(d z),\right. \\
& \left.v \in T M \text { satisfying } \pi(v) \in \bar{B}\left(x_{0}, C(1+C) R\right), F(v)=1\right\}
\end{aligned}
$$

where $v^{N}$ is the normal part of $v$ with respect to the vector $\overrightarrow{\pi(v) z}$ and the scalar product $\langle\cdot, \cdot\rangle_{\overrightarrow{\pi(v) z}}$. If $\eta-\delta>0$, then $\mathscr{F}_{\mu}$ is strictly convex on $\bar{B}\left(x_{0}, C(1+C) R\right)$. More precisely, for all $x \in B\left(x_{0}, C(1+C) R\right)$ and for all unit speed geodesics $\gamma$ starting at $x$,

$$
\left(\mathscr{F}_{\mu} \circ \gamma\right)^{\prime \prime}(0) \geq \eta-\delta .
$$

Proof. With the notation of Section 2, from (2.31) we have

$$
D_{1}^{\prime \prime}(0)=r^{-1}\left(E^{\prime \prime}(0)-\left\langle J^{T}(0), J^{T}(0)\right\rangle_{T(0)}\right) .
$$

Let $\gamma(s)=c(0, s)$, the unit speed geodesic with initial condition $v=J(0)$, and $f(s)=\mathscr{F}_{\mu}(\gamma(s))$. Equation (4.4) together with (2.29) gives

$$
f^{\prime \prime}(0) \geq-\delta+\int_{M} \sqrt{k} \cot (\sqrt{k} \rho(\pi(v), z))\left\langle v^{N}, v^{N}\right\rangle_{\overrightarrow{\pi(v) z}} \mu(d z) \geq \eta-\delta .
$$

From this, we get the condition for the strict convexity of $\mathscr{F}_{\mu}$. 
For $x \in M$, define the measure $\mu_{x}=\mu-\mu(\{x\}) \delta_{x}$. Then the map $y \mapsto \mathscr{F}_{\mu_{x}}(y)$ is differentiable at $y=x$.

Since

$$
\mathscr{F}_{\mu}(y)=\mathscr{F}_{\mu_{x}}(y)+\mu(\{x\}) \rho(y, x)
$$

and, for $v \in T_{x} M, \mathscr{F}_{\mu}$ is differentiable in the direction $v$ with derivative

$$
\left\langle d \mathscr{F}_{\mu}, v\right\rangle=\left\langle d \mathscr{F}_{\mu_{x}}, v\right\rangle+\mu(\{x\}) F(-v),
$$

we see that $x$ is a local minimum of $\mathscr{F}_{\mu}$ if and only if for all non-zero $v \in T_{x} M$,

$$
\mu(\{x\}) F(-v) \geq\left\langle d \mathscr{F}_{\mu_{x}},-v\right\rangle,
$$

which is equivalent to

$$
\mu(\{x\}) \geq \frac{\left(F^{*}\left(d \mathscr{F}_{\mu_{x}}\right)\right)^{2}}{F\left(\mathscr{L}^{-1}\left(d \mathscr{F}_{\mu_{x}}\right)\right)}
$$

(take $\left.-v=\mathscr{L}^{-1}\left(d \mathscr{F}_{\mu_{x}}\right) / F\left(\mathscr{L}^{-1}\left(d \mathscr{F}_{\mu_{x}}\right)\right)\right)$. But, since $F^{*}=F \circ \mathscr{L}^{-1}$, we get the following proposition.

Proposition 4.5. A point $x$ in $M$ is a local minimum of $\mathscr{F}_{\mu}$ if and only if

$$
\mu(\{x\}) \geq F^{*}\left(d \mathscr{F}_{\mu_{x}}\right) .
$$

Note that for the Riemannian case this result is due to Yang [36].

Define the vector

$$
H(x)=\left.\operatorname{grad}_{y}\left(\mathscr{F}_{\mu_{x}}(y)\right)\right|_{y=x}
$$

Alternatively,

$$
H(x)=\mathscr{L}^{-1}\left(\int_{M \backslash\{x\}} \mathscr{L}\left(-\frac{1}{\rho(x, z)} \overrightarrow{x z}\right) \mu(d z)\right) .
$$

Let $a \mapsto x(a)$ be the path in $M$ defined by $x(0)=x_{0}$ and

$$
\dot{x}(a)= \begin{cases}-H(x(a)) & \text { if for all } a^{\prime} \leq a, \mu\left(\left\{x\left(a^{\prime}\right)\right\}\right)<F^{*}\left(d \mathscr{F}_{\mu_{x\left(a^{\prime}\right)}}\right) ; \\ 0 & \text { if for some } a^{\prime} \leq a, \mu\left(\left\{x\left(a^{\prime}\right)\right\}\right) \geq F^{*}\left(d \mathscr{F}_{\mu_{x\left(a^{\prime}\right)}}\right) .\end{cases}
$$

Define

$$
f(a)=\mathscr{F}_{\mu}(x(a)) .
$$

We have for the right derivative of $f$ when $x(a)$ is not a minimal point of $\mathscr{F}_{\mu}$ :

We get

$$
\begin{aligned}
f_{+}^{\prime}(a)= & \left\langle d_{x(a)}\left(\mathscr{F}_{\mu_{x(a)}}\right), \dot{x}(a)\right\rangle+\mu(\{x(a)\}) F(-\dot{x}(a)) \\
= & -\left\langle d_{x(a)}\left(\mathscr{F}_{\mu_{x(a)}}\right), \mathscr{L}^{-1}\left(d_{x(a)}\left(\mathscr{F}_{\mu_{x(a)}}\right)\right)\right\rangle \\
& +\mu(\{x(a)\}) F\left(\mathscr{L}^{-1}\left(d_{x(a)}\left(\mathscr{F}_{\mu_{x(a)}}\right)\right)\right) \\
= & -F^{*}\left(d_{x(a)}\left(\mathscr{F}_{\mu_{x(a)}}\right)\right)^{2}+\mu(\{x(a)\}) F\left(\mathscr{L}^{-1}\left(d_{x(a)}\left(\mathscr{F}_{\mu_{x(a)}}\right)\right)\right) .
\end{aligned}
$$

$$
f_{+}^{\prime}(a)=-F^{*}\left(d_{x(a)}\left(\mathscr{F}_{\mu_{x(a)}}\right)\right)\left(F^{*}\left(d_{x(a)}\left(\mathscr{F}_{\mu_{x(a)}}\right)\right)-\mu(\{x(a)\})\right),
$$

which is negative as soon as $x(a)$ is not a minimal point of $\mathscr{F}_{\mu}$. From this, we get the following proposition.

Proposition 4.6. Assume that $\mu$ is supported by a compact ball $\bar{B}\left(x_{0}, R\right)$, that the support of $\mu$ is not contained in a single geodesic, and that, for all $z \in \bar{B}\left(x_{0}, R\right)$, the forward distance to $z$ is convex, and strictly convex in any geodesic of $\bar{B}\left(x_{0}, C(1+C) R\right)$ which does not contain $z$. Then the path $a \mapsto x(a)$ converges to the median $m$ of $\mu$. 
Proof. This is similar to the proof of Proposition 3.7.

5. An algorithm for computing $p$-means

Lemma 5.1. Assume $\mathscr{K} \geq-\beta^{2}, \mathscr{T} \leq \delta^{\prime}, \mathscr{D} \leq D$ with $\beta>0, \delta^{\prime} \geq 0, D \geq 1$. For $p>1, r>0$, define

$$
H(r)=H_{p, \beta, D, \delta^{\prime}}(r):=p r^{p-2}\left(D^{2} \max ((p-1), r \beta \operatorname{coth}(r \beta))+\delta^{\prime} r\right) .
$$

If $\mu$ is a probability measure on $M$ with bounded support and $x \in M$, define

$$
H_{\mu}(x)=H_{\mu, p, \beta, D, \delta^{\prime}}(x):=\int_{M} H_{p, \beta, D, \delta^{\prime}}(\rho(x, y)) d \mu .
$$

If $t \mapsto \gamma(t)$ is a unit speed geodesic, then, for all $t$,

$$
\left(\mathscr{E}_{\mu, p} \circ \gamma\right)^{\prime \prime}(t) \leq H_{\mu}(\gamma(t))
$$

Proof. For $x, y \in M, r=\rho(x, y), s \mapsto \gamma(s)=c(0, s)$ a unit speed geodesic started at $x=$ $c(0,0)$, and $t \mapsto c(t, s)$ the geodesic satisfying $c(1, s)=y$, we have

$$
D_{p}^{\prime \prime}(0) \leq p r^{p-2}\left(D^{2} \max ((p-1), r \beta \operatorname{coth}(r \beta))+\delta^{\prime} r\right)
$$

Integrating this equation with respect to $y$ gives the result.

REMARK 5.2. If $p \geq 2$ or $\mu$ has a smooth density, then the function $H_{\mu}$ is bounded on all compact sets.

The main result is the following (see [23] for a similar result in a Riemannian manifold).

Proposition 5.3. Assume $-\beta^{2} \leq \mathscr{K} \leq k,-\delta \leq \mathscr{T} \leq \delta^{\prime}, \mathscr{C} \leq C$, and $\mathscr{D} \leq D$ for some $\beta, k, \delta, \delta^{\prime}>0$ and $C, D \geq 1$. Let $p>1$. Assume the support of $\mu$ is contained in $B\left(x_{0}, R\right)$ and $\mathscr{E}_{\mu, p}$ is strictly convex on $\bar{B}\left(x_{0}, C(C+1) R\right)$. Assume furthermore that the function $H_{\mu}=$ $H_{\mu, p, \beta, D, \delta^{\prime}}$ is bounded on $\bar{B}\left(x_{0}, C(C+1) R\right)$ by a constant $C_{H}>0$, and that the injectivity radius at any point of $\bar{B}\left(x_{0}, C(C+1) R\right)$ is larger than $C^{2}+C+1$. Define the gradient algorithm as follows.

Step 1. Start from a point $x_{1} \in B\left(x_{0}, C(C+1) R\right)$ such that $\mathscr{E}_{\mu, p}\left(x_{1}\right) \leq R^{p}$ (take for instance $\left.x_{1}=x_{0}\right)$ and let $k=1$.

Step 2. Let

$$
v_{k}=\frac{\left.\operatorname{grad}\left(-\mathscr{E}_{\mu, p}\left(x_{k}\right)\right)\right)}{F\left(\operatorname{grad}\left(-\mathscr{E}_{\mu, p}\left(x_{k}\right)\right)\right)}, \quad t_{k}=\frac{F\left(\operatorname{grad}\left(-\mathscr{E}_{\mu, p}\left(x_{k}\right)\right)\right)}{C_{H}}
$$

and let $\gamma_{k}$ be the geodesic satisfying $\gamma_{k}(0)=x_{k}, \dot{\gamma}_{k}(0)=v_{k}$. Define

$$
x_{k+1}=\gamma_{k}\left(t_{k}\right)
$$

and then do again Step 2 with $k=k+1$.

Then the sequence $\left(x_{k}\right)_{k \geq 1}$ converges to $e_{p}$.

Proof. We first prove that the sequence $\left(\mathscr{E}_{\mu, p}\left(x_{k}\right)\right)_{k \in \mathbb{N}}$ is non-increasing. For this, we write

$$
\begin{aligned}
\mathscr{E}_{\mu, p}\left(\gamma_{k}\left(t_{k}\right)\right) & \leq \mathscr{E}_{\mu, p}\left(\gamma_{k}(0)\right)+\left\langle d \mathscr{E}_{\mu, p}, v_{k}\right\rangle t_{k}+C_{H} \frac{t_{k}^{2}}{2} \\
& \leq \mathscr{E}_{\mu, p}\left(\gamma_{k}(0)\right)-F\left(\operatorname{grad}\left(-\mathscr{E}_{\mu, p}\left(x_{k}\right)\right)\right) \frac{1}{C_{H}} F\left(\operatorname{grad}\left(-\mathscr{E}_{\mu, p}\left(x_{k}\right)\right)\right)
\end{aligned}
$$




$$
\begin{aligned}
& +\frac{C_{H}}{2}\left(\frac{F\left(\operatorname{grad}\left(-\mathscr{E}_{\mu, p}\left(x_{k}\right)\right)\right)}{C_{H}}\right)^{2} \\
= & \mathscr{E}_{\mu, p}\left(\gamma_{k}(0)\right)-\frac{C_{H}}{2}\left(\frac{F\left(\operatorname{grad}\left(-\mathscr{E}_{\mu, p}\left(x_{k}\right)\right)\right)}{C_{H}}\right)^{2} .
\end{aligned}
$$

This proves that the sequence is non-increasing. As a consequence, for all $k \geq 1, x_{k} \in$ $\bar{B}\left(x_{0}, C(C+1) R\right)$, since $\mathscr{E}_{\mu, p}\left(x_{k}\right) \leq R^{p}$ and, for all $x \notin \bar{B}\left(x_{0}, C(C+1) R\right), \mathscr{E}_{\mu, p}(x)>R^{p}$.

Next we prove that $\mathscr{E}_{\mu, p}\left(x_{k}\right)$ converges to $\mathscr{E}_{\mu, p}\left(e_{p}\right)$. We know that $\mathscr{E}_{\mu, p}\left(x_{k}\right)$ converges to $a \geq \mathscr{E}_{\mu, p}\left(e_{p}\right)$. Extracting a subsequence $x_{k_{\ell}}$ converging to some $x_{\infty} \in \bar{B}\left(x_{0}, C(C+1) R\right)$, this implies that $t_{k_{\ell}}$ converges to 0 . But this is possible only if $x_{\infty}=e_{p}$, which implies that $a=\mathscr{E}_{\mu, p}\left(e_{p}\right)$. As a consequence, any converging subsequence has $e_{p}$ as a limit, and this implies that $x_{k}$ converges to $e_{p}$.

REMARK 5.4. For this result, we need the Hessian of $\mathscr{E}_{\mu, p}$ to be bounded, and the subgradient algorithm in Riemannian manifolds as developed in [36] does not work. The reason is that for this algorithm, we would need to take

$$
v_{k}=\frac{\left.\operatorname{grad}_{\overrightarrow{x_{k} e_{p}}}\left(-\mathscr{E}_{\mu, p}\left(x_{k}\right)\right)\right)}{F\left(\operatorname{grad}_{\overrightarrow{x_{k} e_{p}}}\left(-\mathscr{E}_{\mu, p}\left(x_{k}\right)\right)\right)},
$$

where $\operatorname{grad}_{\overrightarrow{x_{k} e_{p}}}$ denotes the gradient with respect to the metric $\langle\cdot, \cdot\rangle_{\overrightarrow{x_{k}} \vec{e}_{p}}$. So, we would need to know $e_{p}$ !

Corollary 5.5. Let $p=2$. If

$$
R \leq \frac{1}{C(C+1)^{2} \sqrt{k}} \arctan \left(\frac{\sqrt{k}}{C \delta^{2}}\right)
$$

or $M$ has non-positive flag curvature, then the algorithm of Proposition 5.3 can be applied with the appropriate constants.

Proof. With this assumption, by Proposition 2.2 the function $\mathscr{E}_{\mu, 2}$ is strictly convex on $\bar{B}\left(x_{0}, C(C+1) R\right)$.

\section{References}

1. B. Afsari, 'Riemannian $L^{p}$ center of mass: existence, uniqueness, and convexity', Proc. Amer. Math. Soc., S0002-9939(2010)10541-5, Article electronically published on 27 August 2010.

2. J. C. Alvarez Paiva, 'Some problems on Finsler geometry', Handbook of differential geometry II (Elsevier/North-Holland, Amsterdam, 2006) 1-33.

3. S. I. AmARY and H. NAGAOKA, Methods of information geometry, Translations of Mathematical Monographs 191 (American Mathematical Society and Oxford University Press, 2000).

4. M. Arnaudon and X. M. Li, 'Barycenters of measures transported by stochastic flows', Ann. Probab. 33 (2005) no. 4, 1509-1543.

5. L. Astola and L. Florack, 'Finsler geometry on higher order tensor fields and applications to high angular resolution diffusion imaging', Scale Space and Variational Methods in Computer Vision, Second International Conference, Voss, Norway, June 1-5, 2009, Int. J. Comput. Vis. 92 (2011) 325-336.

6. L. Auslander, 'On curvature in Finsler geometry', Trans. Amer. Math. Soc. 79 (1955) no. 2, 378-388.

7. D. BAo, S. S. Chern and Z. Shen, An introduction to Riemann-Finsler geometry, Graduate Texts in Mathematics (Springer, Berlin, 2000).

8. S. S. Chern and Z. Shen, Riemann-Finsler geometry, Nankai Tracts in Mathematics 6 (World Scientific, Singapore, 2005). 
9. J. M. Corcuera and W. S. Kendall, 'Riemannian barycentres and geodesic convexity', Math. Proc. Cambridge Philos. Soc. 127 (1999) no. 2, 253-269.

10. E. Dzhafarov and H. Colonius, Fechnerian metrics in unidimensional and multidimensional stimulus spaces, Psychonomic Bulletin \& Review 6 (Springer, New York, 1999) Issue 2.

11. C. ElkAN, 'Using the triangle inequality to accelerate $k$-means', Proceedings of the Twentieth International Conference on Machine Learning (ICML), Washington, DC, 2003 (AAAI Press, Menlo Park, CA, 2003) 147-153.

12. M. Emery and G. Mokobodzki, 'Sur le barycentre d'une probabilité dans une variété', Séminaire de probabilités XXV, Lecture Notes in Mathematics 1485 (Springer, Berlin, 1991) 220-233.

13. P. T. Fletcher, S. Venkatasubramanian and S. Joshi, 'The geometric median on Riemannian manifolds with application to robust atlas estimation', NeuroImage 45 (2009) S143-S152.

14. B. Fuglede and F. Topsoe, 'Jensen-Shannon divergence and Hilbert space embedding', IEEE Int. Symp. Inf. Theory (2004) 31-31.

15. D. Fuhry, R. Jin and D. Zhang, 'Efficient skyline computation in metric space', Proceedings of the 12 th International Conference on Extending Database Technology: Advances in Database Technology, EDBT'09 (ACM, New York, 2009) 1042-1051.

16. R. Gallego Torrome, 'On the generalization of theorems from Riemannian to Finsler geometry I: metric theorems', arXiv:math/0503704v3 [math.DG] 3 April 2008.

17. V. Garcia and F. Nielsen, 'Simplification and hierarchical representations of mixtures of exponential families', Signal Process. 90 (2010) no. 12, 3197-3212.

18. F. R. Hampel, P. J. Rousseeuw, E. M. Ronchetti and W. A. Stahel, Robust statistics the approach based on influence function (Wiley, New York, 1986).

19. H. KArcher, 'Riemannian center of mass and mollifier smoothing', Commun. Pure Appl. Math. XXX (1977) 509-541.

20. W. S. Kendall, 'Probability, convexity and harmonic maps with small image I: uniqueness and fine existence', Proc. Lond. Math. Soc. (3) 61 (1990) no. 2, 371-406.

21. W. S. Kendall, 'Convexity and the hemisphere', J. Lond. Math. Soc. (2) 43 (1991) no. 3, 567-576.

22. H. W. Kunn, 'A note on Fermat's problem', Math. Program. 4 (1973) 98-107.

23. H. LE, 'Estimation of Riemannian barycentres', LMS J. Comput. Math. 7 (2004) 193-200.

24. J. Melonakos, E. Pichon, S. Angenent and A. Tannenbaum, 'Finsler active contours', IEEE Trans. Pattern Anal. Mach. Intell. 30 (2008) no. 3, 412-423.

25. F. Nielsen and R. Nock, 'Sided and symmetrized Bregman centroids', IEEE Trans. Inf. Theory 55 (2009) no. $66,2882-2904$.

26. S.-I. Онта, 'Uniform convexity and smoothness, and their applications in Finsler geometry', Math. Ann. 343 (2009) 669-699.

27. L. M. Ostresh JR., 'On the convergence of a class of iterative methods for solving the Weber location problem', Oper. Res. 26 (1978) no. 4.

28. M. Péchaud, R. Keriven and M. Descoteaux, 'Brain connectivity using geodesics in HARDI', IEEE International Conference on Medical Image Computing and Computer-Assisted Intervention (MICCAI), London, September 2009, Lecture Notes in Computer Science 5762 (Springer, Berlin, 2009) 482-489.

29. B. Pelletier, 'Informative barycentres in statistics', Ann. Inst. Statist. Math. 57 (2005) no. 4, 767-780.

30. J. PicARD, 'Barycentres et martingales dans les variétés', Ann. Inst. H. Poincaré Probab. Stat. 30 (1994) 647-702.

31. A. SAнiB, 'Espérance d'une variable aléatoire à valeurs dans un espace métrique', Thèse de l'Université de Rouen, 1998.

32. Z. SHEN, 'Riemann-Finsler geometry, with applications to information geometry', Chinese Ann. Math. Ser. B 27 (2006) 73-94.

33. Y. VArdi and C. H. Zhang, 'The multivariate $L_{1}$-median and associated data depth', Proc. Natl. Acad. Sci. USA 97 (2000) 1423-1426.

34. E. Weiszfeld, 'Sur le point pour lequel la somme des distances de $n$ points donnés est minimum', Tôhoku Math. J. 43 (1937) 355-386.

35. B. Y. WU and Y. L. XIN, 'Comparison theorems in Finsler geometry and their applications', Math. Ann. 337 (2007) 177-196.

36. L. YANG, 'Riemannian median and its estimation', LMS J. Comput. Math. 13 (2010) 461-479.

37. C. Zach, L. Shan and M. Niethammer, Globally optimal Finsler active contours, Lecture Notes in Computer Science 5748 (Springer, 2009) 552-561. 
Marc Arnaudon

Laboratoire de Mathématiques et Applications

CNRS: UMR 6086, Université de Poitiers

Téléport 2 - BP 30179, F-86962

Futuroscope, Chasseneuil Cedex

France

marc.arnaudon@math.univ-poitiers.fr
Frank Nielsen

Laboratoire d'Informatique (LIX)

École Polytechnique

91128 Palaiseau Cedex

France

and

Sony Computer Science

Laboratories, Inc

Tokyo

Japan

frank.nielsen@acm.org 